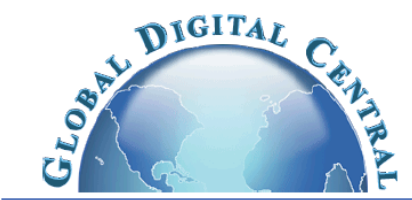

Frontiers in Heat and Mass Transfer

Available at www.ThermalFluidsCentral.org

\title{
A CORRELATION FOR NUSSELT NUMBER UNDER TURBULENT MIXED CONVECTION USING TRANSIENT HEAT TRANSFER EXPERIMENTS
}

\author{
N. Gnanasekaran and C. Balaji* \\ Department of Mechanical Engineering, Indian Institute of Technology Madras, Chennai, India
}

\begin{abstract}
This paper reports the results of an experimental investigation of transient, turbulent mixed convection in a vertical channel in which one of the walls is heated and the other is adiabatic. The goal is to simultaneously estimate the constants in a Nusselt number correlation whose form is assumed a priori by synergistically marrying the experimental results with repeated numerical calculations that assume guess values of the constants. The convective heat transfer coefficient " $h$ "is replaced by the Nusselt number $(\mathrm{Nu})$ and the constants in the Nusselt number are to be evaluated. From the experimentally obtained temperature time history and the simulated temperature time history, based on some guess values of a, b and c, one can define the objective function or the residue as the sum of the square of the difference between experimentally obtained and simulated temperatures. Using Bayesian inference driven by the Markov chain Monte Carlo method, one, more or all of the constants a, b and c are retrieved together with the uncertainty involved in these estimates. Additionally, the estimated parameters are compared with experimental benchmarks.

Keywords: transient, turbulent, markov chain, Bayesian, estimation, correlation.
\end{abstract}

\section{INTRODUCTION}

The estimation of a physical, indirectly, by first measuring the effect(s) and reasoning out the causes invariably through a mathematical model is known as parameter estimation. There could be several causes which cause the same effect(s). Consequent upon this, parameter estimation problems are invariably ill- posed. The estimation can get exceedingly complex and sometimes even tricky, when the mathematical model associated with the phenomenon is highly nonlinear. Parameter estimation is generally done either through deterministic or stochastic models. A gradient based method with an initial guess which is far away from the actual solution invariably fails to retrieve the parameters under question. Stochastic based methods on the other hand do not depend on the initial guess, so are robust and hence are gaining increasing importance in recent times. Consequent upon this, enormous research efforts are being taken to improvise on such methods. The availability of better numerical methods to solve heat transfer problems, better optimization techniques that invariably power the estimation and the availability of faster computers have all contributed to the rapid strides being witnessed in inverse heat transfer.

As aforesaid, a lot of research is being carried out on inverse heat transfer problems and parameter retrieval is becoming increasingly important and relevant. Mixed convection, which incorporates both free convection and forced convection heat transfer, is currently one of the widely researched topics in heat transfer. An early work in mixed convection was due to Lloyd and Sparrow (1969) who studied combined forced and free convection on vertical surfaces. Rao et al. (2002), among others, conducted numerical investigations on conjugate mixed convection with surface radiation from vertical plates and channels and proposed correlations for the average Nusselt number. Krishnan et al. (2004) studied free and mixed convection with surface radiation in isolated and parallel vertical heated plates. Premachandran and Balaji (2006) carried out numerical studies on conjugate mixed convection with and without surface radiation from channels with volumetric heat generation. Tikhnov (1977) and Black et al. (1985) give a comprehensive account of ill-posed inverse problems. Rammohan Rao and Venkateshan (1991) proposed a parameter estimation based on the method of least squares residuals. Venugopal et al. (2008) conducted transient heat transfer experiments and used a hybrid optimization technique to retrieve the constants in a correlation for Nusselt number for laminar mixed convection. Parthasarathy and Balaji (2008) demonstrated the estimation of multiple parameters in two dimensional conduction using Bayesian inference. To the best of the authors knowledge, correlations for turbulent mixed convection, based on parameter estimation with data from transient experiments, with a state- of-theart technique like Bayesian inference has not been reported in literature.

\section{EXPERIMENTAL SETUP}

The test plate consists of a nichrome heater wrapped in a mica sheet and sandwiched between two aluminium plates of $3 \mathrm{~mm}$ thickness. The aluminium plate contains five thermocouples on either side (totally ten thermocouples) cemented with thermal paste along the groove locations. When cooling of the plate takes place the variation in the temperature

${ }^{*}$ Corresponding author. Email: balaji@iitm.ac.in, Phone 91-44-22574689 
has been noticed to vary within $\pm 0.5^{\circ} \mathrm{C}$. Figure. 1 shows the aluminium plate used for the experiments and location of thermocouples. For con-

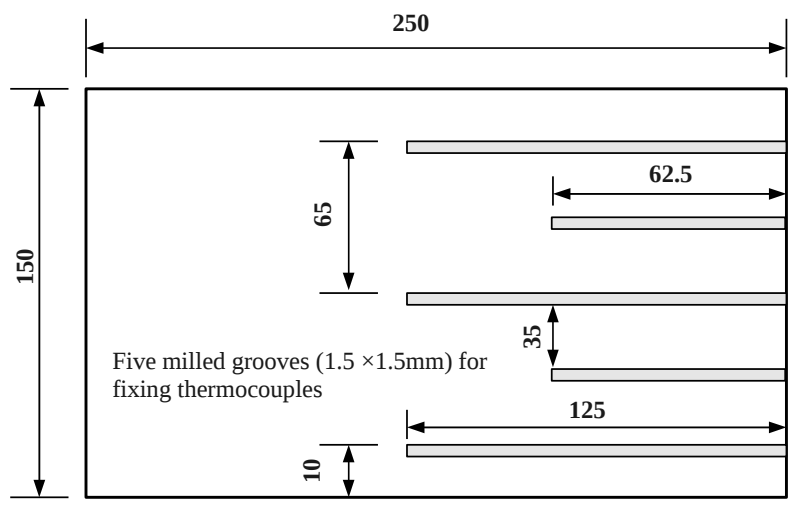

All dimensions are in mm. (dimensions are not to scale)

Fig. 1 Schematic of the aluminium plate

venience and also to exploit the advantage of symmetry, two adiabatic plates are kept on either side of the heater plate assembly in the wooden box at equal distance from the latter. This in a way mimics heat transfer from two similar vertical channels which are shown in Fig.2. The wind

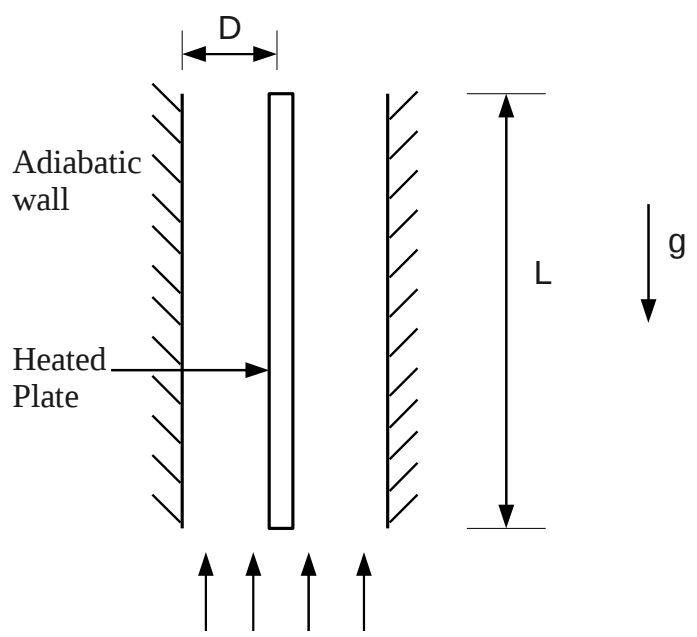

Fig. 2 Schematic of the vertical channel used for the experiments

tunnel shown in Fig. 3 consists of a diffuser, settling chamber, nozzle and a test section. The source of air supplying in the wind tunnel is an axial flow fan mounted below the diffuser. A variable speed motor is used to supply air to the test section to obtain the required velocities. The vertical duct has the following dimensions $390 \times 250 \times 62 \mathrm{~mm}$. Wooden plates made of $12 \mathrm{~mm}$ plywood panels are used to secure the vertical channel to a wooden box which is placed at the top of the wind tunnel. This assembly constitutes the test section. 'L'shaped mild steel brackets are used to fix the wooden plates to each side of the wooden box. Locking nuts are provided such that movement on either side is freely adjusted. Non-rubberized corks are fastened on to two of the wooden plates that constitute the adiabatic walls of the two symmetric channels. To ensure uniform flow a bell mouth is constructed at the inlet of the section. The test section is housed in a low speed vertical wind tunnel that has a capability to provide both assisting and opposing flow.

The experimental procedure involves the transient heating/cooling

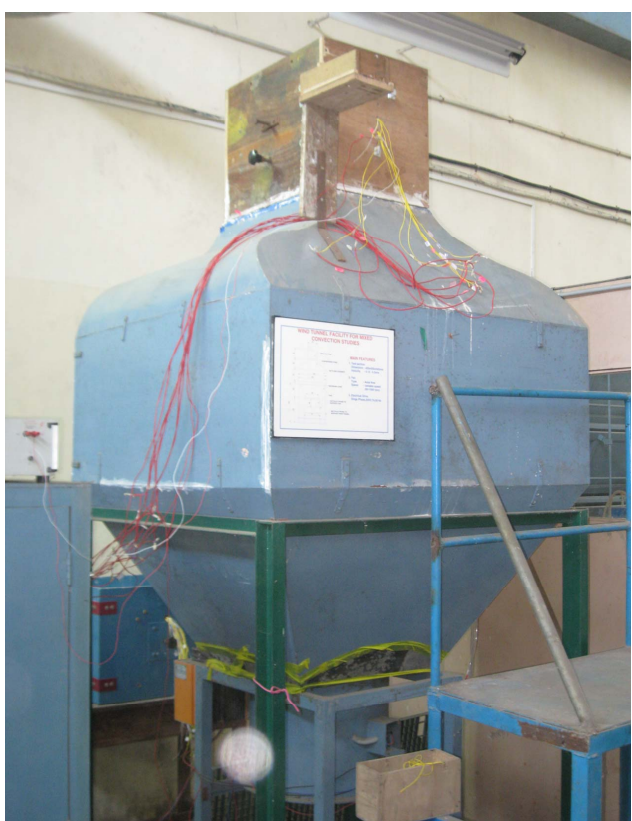

Fig. 3 Schematic of the wind tunnel used for the experiments

of the channels and recording the temperature time history using a data logger. A thermal anemometer which is essentially a thermistor probe, is employed to measure the velocity in the test section and a provision is made in the test section to measure the upstream velocity. High voltage DC power supply can deliver a wide range of power levels. $\mathrm{K}$ type (36AWG) stainless steel sheathed, calibrated thermocouples are provided at various location of the test plate. The salient features of the thermocouples are shown in Table 1. During cooling, for example, the velocity

Table 1 Features of the k-type thermocouple used in the present study.

\begin{tabular}{|c|c|c|}
\hline S.No & \multicolumn{2}{|c|}{ Feature } \\
\hline 1. & Type & K-type (36AWG) \\
\hline 2. & Uncertainty & $0.1 \mathrm{C}$ \\
\hline 3. & Time constant & $3 \mathrm{~s}$ \\
\hline
\end{tabular}

remains fixed and so does the Reynolds number $R e_{D}$. However the average temperature of the heated wall of the channel goes down with time and so does the Grashof number Gr. Hence, during the cooling regime it is possible to experimentally obtain a range of Richardson numbers $\left(R i_{D}\right)$ where $R i_{D}$ is given by $G r_{D} / R e_{D}^{2}$. For the range of temperatures encountered in this study, turbulent mixed convection is expected to take place along the vertical channel.

\subsection{GOVERNING EQUATION AND SOLUTION METHODOLOGY}

The aim of the present study is to use Bayesian inference to obtain a correlation for Nusselt number in mixed convection along a vertical channel, by using data from transient heat transfer experiments in conjunction with Bayesian inference. The mathematical model for the current problem is

$$
E_{\text {stored }}=-E_{\text {lost }}+E_{\text {gen }}
$$

The first term in the right hand side of the above equation is the energy lost due to convection and radiation and the left hand side is the decrease in the energy stored by the system. Incorporating all the necessary terms in "Eq.(1)" for the case of cooling of the plate we get the following equation.

$$
m C_{p} \frac{d T_{h}}{d t}=-\epsilon \sigma A\left(T_{h}^{4}-T_{\infty}^{4}\right)-N u_{D} k_{f} A\left(T_{h}-T_{\infty}\right)
$$


The plate heater assembly is assumed as spatially isothermal. The Biot number $\left(\mathrm{hL}_{c} / \mathrm{k}\right)$ turns out to be less than 0.001 and the lumped formulation is eminently valid, in such a situation. The convective heat transfer in the "Eq.(2)" can be assumed as $N u_{D}=a\left(1+R i_{D}\right)^{b} R e_{D}^{c}$. Further "Eq.(2)" becomes

$$
m C_{p} \frac{d T_{h}}{d t}=-\epsilon \sigma A\left(T_{h}^{4}-T_{\infty}^{4}\right)-a\left(1+R i_{D}\right)^{b} R e_{D}^{c} k_{f} A\left(T_{h}-T_{\infty}\right)
$$

From the above equation the objective of the present work is to retrieve one, more or all of the values $\mathrm{a}, \mathrm{b}$ and $\mathrm{c}$ in the Nusselt number correlation.

"Equation.3" is an initial value problem and is solved by a fourth order Runge-Kuta method by which we can obtain temperature time history. The initial condition is $T_{h}=T_{i}$ at $\mathrm{t}=0$. Starting with guess values of the parameters one can solve "Eq.(3)". This is frequently referred to as the forward model.

\subsection{RETRIEVAL METHODOLOGY}

The temperature time histories numerically obtained by solving the "Eq.(3)" and the experimental temperature time histories have to be matched for the parameter estimation. Bayesian inference is used to estimate the parameters and the general form is given by

$$
P(x / Y)=P(Y / x) \times P(x) / P(Y)
$$

$\mathrm{P}(\mathrm{x} / \mathrm{Y})$ is the posterior probability density function (PPDF) for which ' $\mathrm{X}$ ' is the cause and the effect is ' $Y$ '. For example ' $\mathrm{x}$ ' can be any thermophysical property such as specific heat $\left(C_{p}\right)$, thermal conductivity $(\mathrm{k})$, emissivity $(\epsilon)$, convective heat transfer coefficient (h). 'Y' can be the temperature vector. $\mathrm{P}(\mathrm{Y} / \mathrm{x})$ is the maximum likelihood function which gives the information about the temperature vector (effects) for the corresponding thermo-physical properties (cause). $\mathrm{P}(\mathrm{x})$ is the prior information of the parameter which is to be estimated. Now we are seeking the cause (x) which causes the effect (y). Starting with different initial guess of ' $x$ ' and assuming that the X's are normally distributed among the mean, we can calculate $\mathrm{P}(\mathrm{Y} / \mathrm{x})$ which will be high for those values of $\mathrm{x}$ close to the true value. The probability reduces to values close to zero when $\mathrm{x}$ is far away from the solution. Bayesian inference also helps us to inject a prior if it is known already from previous knowledge and this will hasten the retrieval and also help us in obtaining sharper PPDFs signifying lower standard deviation of the estimates. The PPDF can now be written as

$$
P_{\text {post }}\left(\frac{\phi}{T}\right)=\frac{P_{f}\left(\frac{T}{\phi}\right) P_{p r}(\phi)}{\int P_{f}\left(\frac{T}{\phi}\right) P_{p r}(\phi) d \phi}
$$

where $\phi$ represents the state vector and $\mathrm{T}$ represents the vector of observations (temperatures at various values of time). $\mathrm{P}_{p} r(\phi)$ is the prior probability density function (PDF) of the state $\phi, \mathrm{P}_{f}(\mathrm{~T} / \phi)$ is the conditional probability density function of the measurements given the state vector, and $\operatorname{Ppost}(\phi / \mathrm{T})$ is the posterior probability density function of the state vector.The forward pdfs of the measurement vector are assumed to be normally distributed about the simulated vector for each observation.

$$
P_{f}\left(\frac{\phi}{T}\right)=\frac{1}{\sqrt{2 \pi \sigma^{2}}} \exp \left(\frac{-\left(T_{m e a s}-T_{\text {sim }}\right)^{2}}{2 \sigma^{2}}\right)
$$

In "Eq.(6)" $\sigma$ is the error in the measurement and forward model. From the above equation, it is observed that if the measured values and simulated values match the probability assigned will be more. In general the measured values and simulated values will not agree due to error in the instrument and forward model error. So, the probability will always be less than one. The denominator of "Eq.(5)" is only a normalising constant.

$$
P_{\text {post }}\left(\frac{\phi}{T}\right)=\frac{1}{\left(2 \pi \sigma^{2}\right)^{N / 2}} \exp \left(\frac{-\sum_{i=1}^{N}\left(T_{\text {meas }}-T_{\text {sim }}\right)^{2}}{2 \sigma^{2}}\right)
$$

Where $\mathrm{N}$ is the total number of samples in one single experiment $\chi^{2}$ can now be defined as

$$
\chi^{2}=\sum_{i=1}^{n}\left(\frac{\left.Y_{\text {meas }, i}-Y_{\text {sim }, i}\right)^{2}}{\sigma^{2}}\right.
$$

The Gaussian prior can be given as

$$
P_{p r}(\phi)=\frac{1}{\left(\sqrt{2 \pi \sigma_{p}^{2}}\right)^{n}} \exp \frac{-\left(\phi-\mu_{p}\right)^{2}}{2 \sigma_{p}^{2}}
$$

Upon substituting the above equations, we have

$$
P_{\text {post }}\left(\frac{\phi}{T}\right)=\frac{1}{\left(2 \pi \sigma^{2}\right)^{N / 2}} \exp \left(-\frac{\chi^{2}}{2}\right)+\frac{1}{\left(\sqrt{2 \pi \sigma_{p}^{2}}\right)^{n}} \exp \frac{-\left(\phi-\mu_{p}\right)^{2}}{2 \sigma_{p}^{2}}
$$

Later, knowing all the information about the posterior distribution one can determine the mean and the maximum a posteriori which are given below

$$
\text { Mean }=E\left(P\left(\frac{x}{Y}\right)\right)
$$

The maximum a posteriori is given as

$$
\phi(M A P)=\arg M a x[p(\phi / T)]
$$

\section{STEADY STATE EXPERIMENTS}

First the DC power supply is switched on and a constant power supply is given. The plates get heated up and the spatial variation in the temperature of the plate is obtained as $\pm 0.2^{\circ} \mathrm{C}$ which corroborates our earlier assumption of an isothermal plate. Upon reaching steady state, the following equation holds

$$
Q=-\epsilon \sigma A\left(T_{h}^{4}-T_{\infty}^{4}\right)-a\left(1+R i_{D}\right)^{b} \operatorname{Re}_{D}^{c} k_{f} A\left(T_{h}-T_{\infty}\right)
$$

"Equation.(13)" is the steady state counter part of "Eq.(3)" with an additional heat generation term It can be straightaway used to estimate a,b,c provided enough experiments are conducted. Highly polished aluminium plate whose emissivity is 0.01 was used as the test plate. Limited numbers of steady state experiments were conducted to regress the constants of "Eq.(13)". Based on 19 experiments, the following correlation was obtained.

$$
N u_{D}=0.127\left(1+R i_{D}\right)^{0.728} R e_{D}^{0.644}
$$

"Equation.(14)" has a correlation coefficient of 0.98 and an RMS error of \pm 0.073 . However, it is instructive to mention here that each steady state runs takes about 3 hours. On the contrary, by performing transient experiments, in one unsteady run itself a plethora of measurements are possible. These can then be used in a Bayesian framework to "rapidly"estimate a, b and c. This procedure is elucidated in the ensuing sections.

\section{BAYESIAN BASED MARKOV CHAIN MONTE CARLO (MCMC) ALGORITHM}

The Bayesian based MCMC algorithm proposed in this study has the following steps.

- Specify the number of iterations.

- Guess a value (or randomly choose a number for the existing data vector $\mathrm{x}_{1}$.

- In \#2, $x_{1}$ is the first guess of the data vector. $x_{1}=a$ for the single parameter estimation problem, $\mathrm{x}_{2}=\mathrm{a}, \mathrm{c}$ for the two parameter problem and $\mathrm{x}_{3}=\mathrm{a}, \mathrm{b}, \mathrm{c}$ for the three parameter problem.

- Using a random number and the value of $\mathrm{x}_{1}$, go to $\mathrm{x}_{2}$ (mainly assuming a normal distribution about $\mathrm{x}_{1}$ and a $\sigma$ of $5 \%$ of the current value, $\left.\mathrm{x}_{1}\right)$. 
- Using the forward model obtain the values of Y's for the x's

- Calculate the PPDF for both $\mathrm{x}_{1}$ and $\mathrm{x}_{2}$.

- If $P\left(x_{2}\right)>P\left(x_{1}\right)$, accept the data vector $\mathrm{x}_{2}$.

- Else, calculate the value $\mathrm{Z}=e^{-|\Delta p|}$ and compare $\mathrm{Z}$ with a random number between 0 and 1 .

- If the value is less than a randomly generated $r$, accept else go to step1 with $\mathrm{x}_{2}=\mathrm{x}_{1}$.

The steps 7 and 8 constitute the widely used Metropolis algorithm.

\section{ESTIMATION OF SINGLE PARAMETER}

With the above mentioned procedure, first, a single parameter estimation is done. For this, $\mathrm{b}$ and $\mathrm{c}$ are kept at values obtained from "Eq.(3)". The constant ' $a$ ' is now determined using the Markov Chain Monte Carlo approach.

\section{ESTIMATION OF MULTIPLE PARAMETERS}

The simultaneous retrieval of two/all three parameters is also possible with the help of MCMC and this is the major novelty of this paper. The important point incorporated in the transient model is the study of the assumption of the thermal equilibration of the plate, as heat loses takes place from the plate it is almost instantaneous. Such an assumption is truly valid for materials having high thermal conductivity and that have a reasonable thickness. The quasisteady assumption adopted in this study supposes that the thermal layer passes through a succession of instantaneous steady states. With the values obtained from steady state experiments, the range of the parameters are well known and one unsteady experiment is used retrieve the values of 'a', 'b' and 'c'by injecting an appropriate priors. In this case, Gaussian priors with means corresponding to steady state estimates of parameters a, b, c and appropriate values of standard deviation are used.

\section{RESULTS AND DISCUSSION}

Table 2 shows the mean, MAP and SD for single parameter estimation. Figure.4 shows the results of the single parameter retrieval constant ' $a$ ', for a sample size of 10000 . The burn-in is taken to be 2000. Stated explicitly, the first 2000 values of the PPDF are not used to compute the mean and MAP, in order to remove any bias caused by the initial guess values.

Table 2 Mean, MAP, SD for constant 'a'- single parameter estimation

\begin{tabular}{|c|c|c|c|c|}
\hline S.NO & Power & Mean & MAP & SD \\
\hline 1. & 38.7 & 0.118 & 0.130 & 0.036 \\
\hline 2. & 49.0 & 0.130 & 0.140 & 0.039 \\
\hline 3. & 109.5 & 0.170 & 0.170 & 0.029 \\
\hline \multicolumn{2}{|c|}{ Avg } & 0.139 & 0.146 & 0.035 \\
\hline
\end{tabular}

Similarly the value for ' $c$ ' is determined to be 0.686 keeping a and b fixed at values obtained from Eq. (14). Table 3 shows the mean, MAP and standard deviation in the estimates of the parameters for three different power levels for the estimation of constant 'c'in the Nusselt number correlation. Figure. 5 shows the typical PPDF for the constant 'c'in the Nusselt number correlation.

Table 4 shows the mean and standard deviation of the Gaussian priors used for the simultaneous retrieval of a, b, c. As afore mentioned, $\mu_{p}$ values are based on estimates from the steady state experiments.

Table 5 shows the results of a simultaneous retrieval of constants a, $\mathrm{b}$ and $\mathrm{c}$ in the Nusselt number correlation. The simultaneous retrieval of the constant 'a ', 'b 'and 'c 'with help of MCMC driven algorithm gives

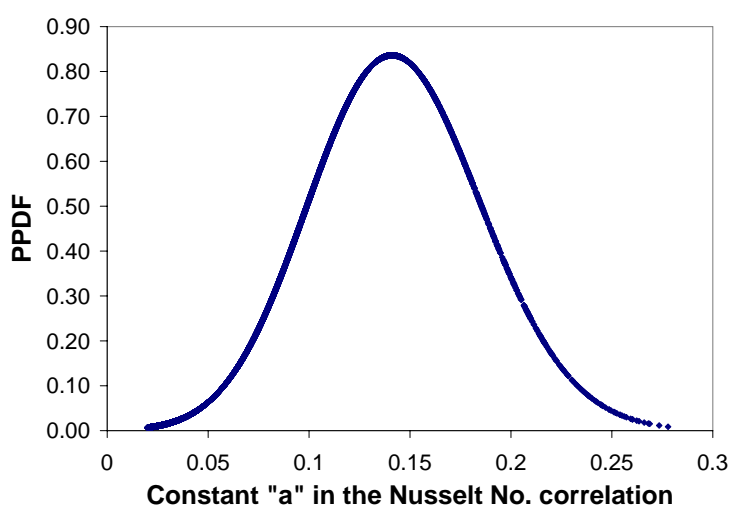

Fig. 4 PPDF of constant ' $a$ ' in the Nusselt number correlation $(38.7 \mathrm{~W})$

Table 3 Mean, MAP, SD for constant 'c'- single parameter estimation

\begin{tabular}{|c|c|c|c|c|}
\hline S.NO & Power & Mean & MAP & SD \\
\hline 1. & 38.7 & 0.650 & 0.660 & 0.060 \\
\hline 2. & 49.0 & 0.660 & 0.67 & 0.037 \\
\hline 3. & 109.5 & 0.680 & 0.689 & 0.400 \\
\hline \multicolumn{2}{|c|}{ Avg } & 0.686 & 0.686 & 0.0016 \\
\hline
\end{tabular}

us not only the retrieved values but also the standard deviation of the estimates as is the case with the single parameter estimation. Figure 6,7 and 8 show the typical PPDF of retrieved parameters. The retrieved values using Bayesian driven MCMC method show remarkable consistency regardless of the power levels. From Table 5 it is seen that the values of constants 'a ', 'b ' and 'c 'turn out to be $0.127,0.725$ and 0.678 respectively and are in reasonably good agreement with the values obtained from the steady state experiments. The correlation turns out to be

$$
N u_{D}=0.127\left(1+R i_{D}\right)^{0.725} R e_{D}^{0.678}
$$

The estimates a,b,c are valid for $1400 \leq R e_{D} \leq 20000$ and $0.00037 \leq$ $R i_{D} \leq 0.712$. The prior probability $\left(p_{p r}\right)$ for the parameters 'a', 'b' and 'c'are shown in the Table.5. It can be seen that the S.D of the estimates in Table. 4 are much smaller compared to those of the priors (Table 4 column 4) thereby reconfirming with the data, we can get much better estimates compared to the priors.

The excellent agreement between Eqs. 14 and 15 corroborates the efficacy of generating a Nusselt number correlation from transient experiments. Additional steady state experiments were also done and Nusselt number obtained from these (which were not used for obtaining Eq.14) were compared with estimate from Eq.15 and the agreement was found to be reasonable $( \pm 15 \%)$.

\section{CONCLUSIONS}

Transient heat transfer turbulent mixed convection experiments have been done for a highly polished vertical aluminum plate, to retrieve multiple parameters by using Bayesian inference. The transient temperature time history is used to estimate the constants of the Nusselt number correlation. For doing this, only the general form of the correlation needs to be assumed. Using the Bayesian frame work, with the Markov Chain and Monte Carlo method for sampling these constants, it is possible to retrieve the constants rapidly and accurately. The concept of instantaneous Richardson number is made use of here and one unsteady run gives us a range of Richardson numbers. In summary, this study opens up new 


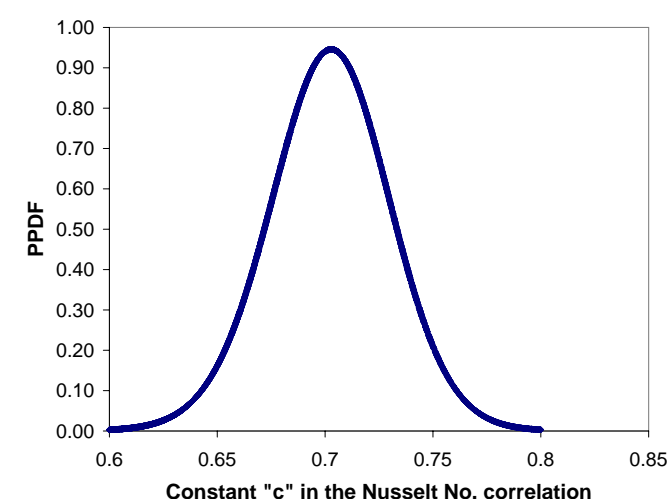

Fig. 5 PPDF of constant 'c'in the Nusselt number correlation (38.7W)

Table 4 Gaussian prior for Multiple parameter estimation

\begin{tabular}{|c|c|c|c|}
\hline S.NO & Parameters & $\mu_{p}$ (Mean of prior) & $\sigma_{p}$ (SD of prior) \\
\hline & $\mathrm{a}$ & 0.127 & 0.015 \\
1. & $\mathrm{~b}$ & 0.728 & 0.10 \\
& $\mathrm{c}$ & 0.644 & 0.10 \\
\hline
\end{tabular}

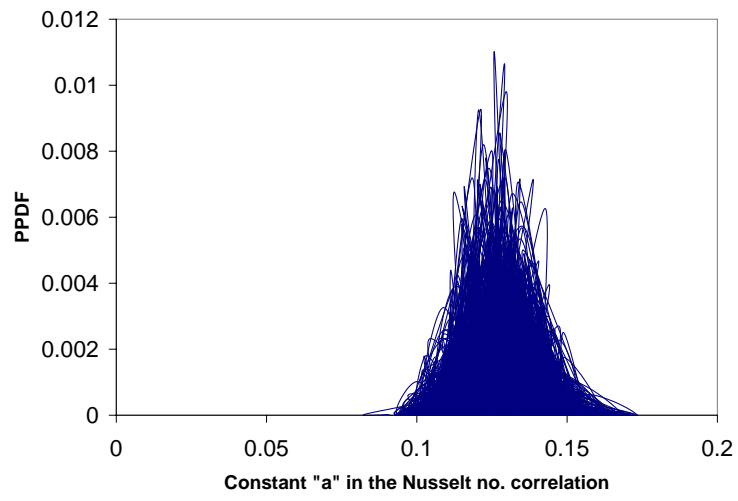

Fig. 6 PPDF of constant $a$ in the Nusselt number correlation (38.7W)

vistas for developing Nusselt number correlations from temperature-time histories that drive a Bayesian based Markov Chain Monte Carlo method. Limited steady state experiments can be used to generate appropriate priors that can be injected in to the Bayesian framework for obtaining radically improved Nusselt number correlations with substantial reductions in the standard deviations of the estimates.

\section{NOMENCLATURE}

$A \quad$ surface area of the test plate, $\left(\mathrm{m}^{2}\right)$

$a, b, c \quad$ constants in the Nusselt number correlation

$C_{p} \quad$ specific heat capacity of the test plate, $(\mathrm{kJ} / \mathrm{kg} \mathrm{K})$

$D \quad$ spacing of the parallel plate channel, (m)

$G r_{D} \quad$ Grashof number based on the spacing of the channel,

$h \quad$ convective heat transfer coefficient, $\left(\mathrm{W} / \mathrm{m}^{2} \mathrm{~K}\right)$
Table 5 Mean, MAP and SD- Multiple parameter estimation

\begin{tabular}{|c|c|c|c|c|c|}
\hline S.NO & Power, $W$ & Constants & Mean & MAP & $S D$ \\
\hline \multirow{3}{*}{1.} & \multirow{3}{*}{38.7} & $\mathrm{a}$ & 0.127 & 0.125 & 0.008 \\
\hline & & b & 0.723 & 0.722 & 0.050 \\
\hline & & $\mathrm{c}$ & 0.660 & 0.660 & 0.040 \\
\hline \multirow{3}{*}{2.} & \multirow{3}{*}{49.0} & $a$ & 0.127 & 0.127 & 0.008 \\
\hline & & b & 0.726 & 0.710 & 0.048 \\
\hline & & $\mathrm{c}$ & 0.685 & 0.680 & 0.042 \\
\hline \multirow{3}{*}{3.} & \multirow{3}{*}{109.5} & $\mathrm{a}$ & 0.127 & 0.129 & 0.008 \\
\hline & & b & 0.727 & 0.720 & 0.046 \\
\hline & & $\mathrm{c}$ & 0.690 & 0.710 & 0.030 \\
\hline \multirow{3}{*}{\multicolumn{2}{|c|}{ Avg }} & $\mathrm{a}$ & 0.127 & 0.127 & 0.008 \\
\hline & & b & 0.725 & 0.717 & 0.048 \\
\hline & & $\mathrm{c}$ & 0.678 & 0.683 & 0.037 \\
\hline
\end{tabular}

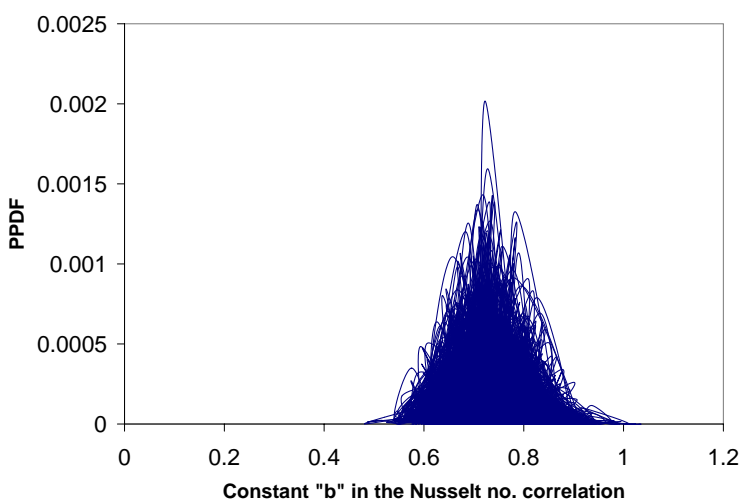

Fig. 7 PPDF of constant $b$ in the Nusselt number correlation (38.7W)

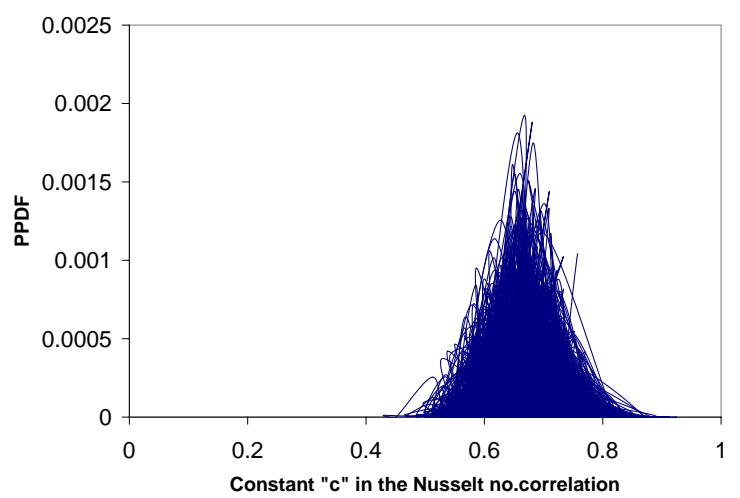

Fig. 8 PPDF of constant $c$ in the Nusselt number correlation $(38.7 \mathrm{~W})$

$k_{f} \quad$ thermal conductivity of the fluid, (W/mK)

$L_{c} \quad$ characteristic length,(m)

$M A P \quad$ maximum a posteriori

$N u_{D} \quad$ Nusselt number, (h L/D)

$P P D F \quad$ posterior probability density function

$\operatorname{Re}_{D} \quad$ Reynolds number based on spacing of the channel, $(D U / \nu)$ 
$R i_{D} \quad$ Richardson number based on spacing of the channel, $\left(G r_{D} / R e_{D}^{2}\right)$ Flow on Vertical Surfaces,"International Journal of Heat Mass Transfer,

$S D \quad$ standard deviation

$t \quad$ time, $(\mathrm{s})$

$T_{i} \quad$ initial temperature, $(\mathrm{K})$

$T_{\infty} \quad$ ambient temperature, $(\mathrm{K})$

Greek Symbols

$\epsilon \quad$ hemispherical total emissivity

$\sigma \quad$ Stefan-Boltzmann constant $\left(\mathrm{W} / \mathrm{m}^{2} \cdot \mathrm{K}^{4}\right)$

Subscripts

I initial

$h \quad$ hot test plate

meas measured

T temperature

\section{REFERENCES}

Beck, J.V., Blackwell, B., and St. Clair, Jr., C.R., 1985, Inverse Heat Conduction-Ill posed problems, Wiley, New York,

Gururaja Rao, Balaji, C, Venkateshan, S. P., 2002, "Effect of Surface Radiation on Conjugate Mixed Convection in a Vertical Channel with a Discrete Heat Source in Each Wall, "International Journal of Heat and Mass Transfer, Vol. 45, pp. 3331 - 3347.

Krishnan, A.S., Balaji, C., Venkateshan, S.P., 2004, “An Experimental Correlation for Combined Convection and Radiation between Parallel Vertical Plates, "ASME, Journal of Heat Transfer, Vol. 126, pp. 849 - 851.

http://dx.doi.org/10.1115/1.1795245

Lloyd J.R, Sparrow E.M., 1969, "Combined Forced and Free Convection
Vol. 12, pp. 434 - 438.

Premachandran, B., Balaji, C., 2006, "Conjugate Mixed Convection with Surface Radiation from a Horizontal Channel with Protruding Heat Sources," International Journal of Heat and Mass Transfer, Vol. 49, pp. 3568 3528.

http://dx.doi.org/10.1080/10407782.2011.588577

Parthasarathy S. and Balaji, C., 2008, "Estimation of Parameters in MultiMode Heat Transfer Problems Using Bayesian Inference - Effect of Noise and a Priori, "International Journal of Heat and Mass Transfer, Vol. 51, pp. $2313-2334$.

http://dx.doi.org/10.1016/j.ijheatmasstransfer .2007 .08 .031

Rammohan Rao, V., and Venkateshan, S. P., 1991, "Parameter EstimationImbedding Technique Verses Least Square Residual Method," Heat and Mass Transfer, Proceedings of the 11th National Heat and Mass Transfer Conference, pp. 303 - 308.

Tikhonov., A.N., 1977, Solution of Ill-posed Problems, Halsted Press, Washington.

Venugopal, G., Balaji, C., Venkateshan, S. P., 2008, "A Correlation for Laminar Mixed Convection from Vertical Plates Using Transient Experiments," Journal of Heat Mass Transfer, Vol. 44, pp . 1417 - 1425.

http://dx.doi.org/10.1007/s00231-008-0380-x 